\title{
Aortic Stiffness and Heart Failure in Chronic Kidney Disease
}

\author{
Monika Gawor ${ }^{1,2} \cdot$ Valentina O. Puntmann ${ }^{2,3}$
}

Published online: 22 April 2020

(C) The Author(s) 2020

\begin{abstract}
Purpose of Review To provide an update on the recent findings in the field of aortic stiffness and heart failure in patients with chronic kidney disease (CKD).

Recent Findings Stratification of cardiovascular risk in CKD remains an open question. Recent reports suggest that aortic stiffness, an independent predictor of cardiovascular events in many patient populations, is also an important prognostic factor in CKD. Also, novel measures of myocardial tissue characterization, native T1 and T2 mapping techniques, have potential as diagnostic and prognostic factors in CKD.

Summary Cardiovascular magnetic resonance has the ability to thoroughly evaluate novel imaging markers: aortic stiffness, native T1, and native T2. Novel imaging markers can be used for diagnostic and prognostic purposes as well as potential therapeutic targets in CKD population.
\end{abstract}

Keywords Aortic stiffness $\cdot$ Heart failure $\cdot$ Chronic kidney disease $\cdot$ Cardiovascular magnetic resonance

\section{Introduction}

Chronic kidney disease (CKD) is associated with high prevalence of cardiovascular (CV) diseases, including heart failure (HF) $[1,2]$. CKD is present in approximately one-third of patients with HF [3]. According to the latest reports, the prevalence of $\mathrm{CKD}$ in $\mathrm{HF}$ might be even higher, ranging from 50 to $63 \%[4,5]$. Presence of both diseases, HF and CKD, is strongly related to worse prognosis, including increased risk of hospitalization, all-cause mortality, and CV mortality [4-6,7•].

The relationship between CKD and HF is bidirectional and complex. HF is a known risk factor for the development of CKD

This article is part of Topical Collection on Cardiac Magnetic Resonance

Monika Gawor

mgawor@ikard.pl

1 Department of Cardiomyopathy, The Cardinal Stefan Wyszynski National Institute of Cardiology, Warsaw, Poland

2 Institute of Experimental and Translational Cardiac Imaging, DZHK Centre for Cardiovascular Imaging, Goethe University Hospital Frankfurt, Frankfurt am Main, Germany

3 Department of Cardiology, Goethe University Hospital Frankfurt, Frankfurt am Main, Germany leading to the hemodynamic changes, neurohormonal activation, endothelial injury, and inflammation that may further injure the kidney function [8]. On the other hand, renal failure is one of the non-cardiovascular causes of HF associated with water retention, electrolyte perturbations, and neurohormonal abnormalities resulting in volume overload, hypertension, abnormal vascular calcification, and advanced myocardial remodeling $[1,9]$. Renal failure is responsible for the progression of $\mathrm{HF}$ and poorer outcomes in this population [10]. According to the recent findings from Chronic Renal Insufficiency Cohort Study, there are several factors, besides decreasing estimated glomerular filtration rate (eGFR), which are independently associated with increased risk of developing $\mathrm{HF}$ in patients with $\mathrm{CKD}$, such as anemia, albuminuria, insulin resistance, inflammation, and poor glycemic control [11]. To complicate things further, not only the prognosis is worse in the presence of both HF and CKD but also therapy of such patients is more demanding $[9,12]$. HF patients with CKD require more caution because of the higher risk of drug toxicity. Treatment of these comorbidities is based on the limited direct evidence of therapy safety and efficacy due to little number of trials conducted in this particular population. Moreover, renal failure is rarely taken into consideration as a therapeutic target in HF, although such an approach could improve patient prognosis $[13,14]$.

Currently, stratification of CV risk in CKD patients is based on eGFR level. Patients with stage $3 \mathrm{CKD}$ already belong to "high CV risk" group and upgrade to "very high CV risk" 
group, if CKD aggravates to stage 4 or 5 [15]. Taking into consideration the fact that there is no consensus on the most reliable measure of eGFR calculation, the assessment of CV risk in CKD based on eGFR level seems inaccurate [11]. In addition, the restoration of kidney function after renal transplantation reduces, but does not eliminate increased CV risk, which still remains much higher than in general population [16-18]. This finding further challenges the appropriateness of using eGFR-based risk stratification. The increased CV risk in CKD patients cannot be also explained only by the presence of traditional CV risk factors, such as diabetes mellitus, dyslipidemia, hypertension, and atherosclerosis [15]. All these facts make the stratification of CV risk in CKD an open question. Recent reports suggest that aortic stiffness, an independent predictor of adverse $\mathrm{CV}$ events in many patient populations, is an important prognostic factor also in CKD patients, associated with left ventricular (LV) remodeling, HF and worse prognosis $\left[15,19,20^{\bullet}\right]$. Also novel measures of myocardial tissue characterization, native T1 and T2 mapping techniques, have potential as diagnostic and prognostic factors in $\operatorname{CKD}[21 \bullet, 22,23 \bullet]$.

\section{Cardiac Involvement in CKD}

Myocardial involvement in the presence of CKD consists of diastolic dysfunction, impaired LV and right ventricular (RV) systolic longitudinal function, as well as adverse remodeling including LV hypertrophy, fibrosis, and ventricular dilatation. These abnormalities are often accompanied by arterial stiffness, accelerated atherosclerosis, valvular calcification, and coronary artery calcification leading to further pathologies: heart valve dysfunction and coronary artery disease complications [24•, 25-27].

The novel findings confirm the importance of the interaction between the kidney and the heart, showing that even patients in the very early stages of CKD are developing cardiac structural and functional abnormalities [28]. One of the earliest signs of subclinical cardiac involvement of CKD is increased LV mass and increased aortic stiffness [29, 30•]. It was recently demonstrated in the group of living kidney donors - healthy subjects, who underwent unilateral nephrectomy. Of note, no progression of atherosclerosis was observed in this study during 1-year follow-up [30•]. Results of another research also indicate strong association of renal function loss with cardiovascular remodeling. LV hypertrophy, predominantly concentric type, occurs in over half of patients with stage $3 \mathrm{CKD}$ and even more frequently among patients with CKD 4 stage [24•]. Interestingly, renal transplant recipients have a higher incidence of cardiovascular remodeling than patients with CKD 3 and lower than patients with CKD 4 stage, showing again incomplete regression of LV hypertrophy and valve and vascular calcification after kidney transplantation [24•]. Presence of LV hypertrophy and fibrosis in CKD patients may explain higher rates of recurrent ventricular tachyarrhythmias, appropriate device therapies, and increased all-cause mortality reported recently in ICD recipients from the CKD population [31].

\section{Cardiovascular Magnetic Resonance in CKD}

Cardiac involvement in CKD can be thoroughly evaluated using cardiovascular magnetic resonance (CMR). CMR provides accurate, reproducible assessment of LV and RV structure and function. At the same time, CMR has the ability to non-invasively evaluate pathological myocardial processes using native $\mathrm{T} 1$ and $\mathrm{T} 2$ mapping techniques - novel quantitative tissue characterization measures of non-infarcted myocardium [32]. CMR enables also to calculate central aortic pulse wave velocity (PWV), a measure of aortic stiffness [20 ]. Notably, the evaluation of novel imaging markers, native T1 and T2, as well as PWV, is independent of the use of gadolinium-based contrast agents (GBCA). Risk of nephrogenic systemic sclerosis (NSF), the use of GBCA in patients with CKD disease was controversial for a long time, especially in severe CKD [33]. However, the current state of the knowledge and formal recommendations for minimizing the risk of NSF allow the diagnostic use of macrocyclic agents in the lowest possible dose across all CKD stages [33-35]. Hemodialysis patients undergo dialysis on the same day a CMR scan with GBCA was performed.

The change in the approach to using GBCA in CKD is important for several reasons. Firstly, the use of GBCA enables the visualization of myocardial replacement fibrosis, which corresponds to the areas of late gadolinium enhancement (LGE) and allows excluding these regions from the measurements of diffuse myocardial fibrosis by T1 mapping [35]. Although in CKD native T1 values are elevated regardless of the presence of myocardial ischemia or post-infarction scar, it is important to separate LGE from T1 mapping region of interest, because it may falsely increase the results of the measurements $[23 \cdot, 32,36]$. Secondly, the possibility of using CMR with LGE imaging and stress perfusion imaging to assess ischemic heart disease is extremely beneficial in the CKD population, which is at increased risk for atherosclerosis [32, 35]. According to the recent findings, perfusion-CMR has strong prognostic value for ischemia detection and a high concordance with invasive coronary angiography combined with fractional flow reserve measurement (FFR) [35]. Notably, perfusion-CMR with macrocyclic contrast agents, compared with invasive coronary angiography or computed tomography coronary angiography, is safer in CKD patients and can diagnose, in addition to ischemic heart disease, other pathologies responsible for chest pain: myocarditis, pericarditis, or microvascular disease [32]. Moreover, it turned out that 
patients with CKD had higher incidence of non-cardiac findings, which are clinically significant in approximately $15 \%$ and can be reliably diagnosed using CMR imaging [37॰].

\section{T1 and T2 Mapping Techniques in CKD}

Native T1 is a non-invasive imaging marker of myocardial abnormalities related to the presence and severity of myocardial edema, diffuse fibrosis, inflammation, or infiltration [32, 36]. Native $T 2$ is water-specific and reflects excess of myocardial fluid, which co-localizes with myocardial inflammation, edema, or injury [32]. Several studies demonstrated higher native $\mathrm{T} 1$ values in $\mathrm{CKD}$ comparing with controls. In addition, native $\mathrm{T} 1$ values range from early-stage to end-stage renal failure $[21 \bullet, 22,23 \bullet]$. Similarly to healthy volunteers, hemodialysis patients have significantly higher native $\mathrm{T} 1$ in interventricular septum than in non-septal myocardium [22]. Increased native $\mathrm{T} 1$ values correlate positively with $\mathrm{LV}$ mass indexed to body surface area and end-diastolic volume in hemodialysis patients $[22,38]$. Native $\mathrm{T} 1$ seemed to be reproducible and unaffected by the changes in fluid status, provided that patients are scanned in the standardized way (e.g., on a day between their dialysis sessions). There was no significant correlation between change in native $\mathrm{T} 1$ values and change in body weight of hemodialysis patients, who underwent repeated CMR scan at a fixed interval from dialysis [39•]. However, another study showed changes in native $\mathrm{T} 1$ and native $\mathrm{T} 2$ in patients who underwent pre- and post-dialysis CMR scans at short intervals [40]. The mean time between the first and second CMR scan was $9.1 \pm 1.1 \mathrm{~h}$, and mean time from completion of dialysis to the second scan was $3.5 \pm 1.3 \mathrm{~h}$. Both native T1 and native T2, as well as indexed LV mass, were significantly lower post-hemodialysis. Good correlation between change in indexed LV mass and change in body weight was found. Interestingly, significant improvement in systolic function was observed post-hemodialysis in a group of patients with impaired LV function (LVEF < 45\%) [40]. These findings reflect the effect of reduction in myocardial water content following hemodialysis. The results also support the thesis that native $\mathrm{T} 1$ is related to myocardial edema, which, if chronic, leads to interstitial fibrosis. The relation between native T1 and markers of adverse LV remodeling in CKD supports the thesis that the mechanism of cardiac dysfunction in CKD is not a consequence of potential ischemic heart disease, but results from the diffuse non-ischemic myocardial changes. The findings from these studies underline also the importance of performing CMR scans at the same timepoint in the weekly dialysis schedule when tracking longer-term changes [40].

Recent study demonstrated that $\mathrm{T} 1$ and T2 mapping may distinguish myocardial involvement in CKD from the phenotypically similar presentations in hypertrophic cardiomyopathy $(\mathrm{HCM})[41 \bullet]$. Native T1 identified abnormal myocardium underlying the hypertrophic remodeling in both pathologies; however, native $\mathrm{T} 2$ values were increased only in the CKDdriven hypertrophic changes [41•]. The results of another study showed increased values of natives $\mathrm{T} 1$, reduction of $L V$ volumes, and no change in $L V$ mass and natives $T 2$ values in patients 2 months after renal transplantation [42]. Slow improvement in cardiac function after renal transplantation, which was reported in several studies, is in line with previous findings that renal failure not only is a prognostic marker in HF but also triggers the HF progression.

Knowing the fact that reversal of cardiac abnormalities after renal transplant is slow and incomplete, early detection of cardiac impairment and rapid therapy implementation are crucial for improving the clinical outcomes in CKD patients $[24 \cdot, 42-44]$. Potentially, native T1 could be helpful as a marker of early myocardial changes in CKD and used for guiding anti-remodeling therapy. Making native T1 reduction a potential therapeutic target would provide means to modify $\mathrm{CV}$ risk and improve the morbidity and mortality of CKD patients.

\section{Aortic Stiffness}

Aortic stiffness improves CV risk classification in numerous subpopulations including patients with CKD $[15,20 \bullet]$. According to the Multi-Ethnic Study of Atherosclerosis (MESA), significantly increased aortic stiffness is associated with LV remodeling and reduced LV systolic and diastolic function [45•]. Moreover, aortic stiffness is independently related to LV remodeling and the presence of diffuse myocardial fibrosis evaluated by T1 mapping in patients with dilated cardiomyopathy [46]. Novel findings suggest that aortic stiffness is one of the earliest signs of subclinical cardiac involvement, connected to the increased $\mathrm{CV}$ risk and myocardial remodeling also in the CKD patients $[20 \bullet, 30 \bullet, 47 \bullet, 48]$. Aortic distensibility (AD) is decreased in hemodialysis patients, while PWV is significantly increased in this population [48]. Moreover, aortic stiffness evaluated 3 months after renal transplantation was still a strong independent predictor of mortality. In addition, no significant changes in aortic stiffness were observed in the first year after renal transplantation [47•].

There are several different tools to non-invasively assess PWV, including tonometric and oscillometric methods as well as CMR-based techniques [49, 50]. Many studies focus on comparing different approaches to PWV assessment and try to determine the simplest and most reliable methods that could be helpful in everyday practice $[49,51,52]$. Recent research, dedicated to comparing different CMR-based techniques of aortic stiffness evaluation in hemodialysis patients, demonstrated excellent inter- and intra-observer variability of ascending and descending AD as well as aortic PWV measurements. The study did not show any significant correlations between inter-study changes in markers of cardiac loading 
and hydration status as well as in aortic stiffness measures; provided that patients are scanned under the standardized conditions. CMR-derived measures of aortic stiffness seem to be reproducible and unaffected by the changes in fluid status. All three parameters of aortic stiffness were associated in this study with indexed LV mass on univariate analysis [53].

The previous literature data on aortic stiffness reflects recently reported findings of a prospective, observational study, conducted to comprehensively characterize CVD in CKD using CMR imaging markers: PWV and native T1 [23•]. The study shows strong relation between aortic stiffness and myocardial hypertrophic-fibrotic remodeling in CKD. There was no such relation in the non-CKD cohort despite similar $\mathrm{CV}$ risk profile as CKD patients. Aortic stiffness and native T1 were significantly higher and strongly related to eGFR in CKD group. The association between PWV and native T1 was amplified with increasing severity of CKD [23•]. More recently, Winau et al [54•] showed an important link between myocardial inflammation and injury, which is amplified through increased PVW. Using CMR with $\mathrm{T} 1$ and $\mathrm{T} 2$ mapping and PWV in patients with systemic inflammatory disease, myocardial T2 mapping measurements were the strongest predictor of hs-TropT release, underscoring the myocardial inflammation as the main mechanism of injury. Patients with active inflammation (by raised T2 values) had much stronger association between native $\mathrm{T} 1$ and PWV. On the contrary, those without active myocardial inflammation (by normal $\mathrm{T} 2$ values) showed diffuse fibrosis by native T1. These findings substantiate the role of CMR in screening of subclinical cardiac involvement. This evidence is important for several reasons. First of all, it highlights the prominent role of native $\mathrm{T} 1$ in an ongoing myocardial injury, leading to functional impairment and consequently, worse prognosis in CKD. Secondly, it supports the established concept of aortoventricular interdependence, postulating adverse myocardial remodeling, including hypertrophy and fibrosis as a consequence of the increased aortic stiffness and LV afterload [45•, 46]. It is therefore possible that aortic stiffness assessment can be used as an early biomarker for $\mathrm{CV}$ dysfunction in patients with $\mathrm{CKD}$. Moreover, PWV and native T1, as sensitive measures of adverse myocardial remodeling, might be useful in selecting CKD patients, who would most likely benefit from therapy optimization, possibly before the onset of first symptoms and development of overt HF.

\section{Conclusions}

Earlier detection of myocardial abnormalities, implementation of the proper treatment, and accurate assessment of $\mathrm{CV}$ risk are necessary steps to prevent development of HF in CKD. The novel imaging markers: aortic stiffness, native T1, and native $\mathrm{T} 2$ are useful diagnostic and prognostic tools in CKD, which can be thoroughly evaluated by CMR imaging. Making aortic stiffness, native $\mathrm{T} 1$, and native $\mathrm{T} 2$ potential therapeutic targets would provide means to modify CV risk and improve the morbidity and mortality of CKD patients.

Funding Information MG was supported by European Association of Cardiovascular Imaging (EACVI) Research Grant 2019.

\section{Compliance with Ethical Standards}

Conflict of Interest All authors declare no conflict of interest.

Human and Animal Rights and Informed Consent This article does not contain any studies with human or animal subjects performed by any of the authors.

Open Access This article is licensed under a Creative Commons Attribution 4.0 International License, which permits use, sharing, adaptation, distribution and reproduction in any medium or format, as long as you give appropriate credit to the original author(s) and the source, provide a link to the Creative Commons licence, and indicate if changes were made. The images or other third party material in this article are included in the article's Creative Commons licence, unless indicated otherwise in a credit line to the material. If material is not included in the article's Creative Commons licence and your intended use is not permitted by statutory regulation or exceeds the permitted use, you will need to obtain permission directly from the copyright holder. To view a copy of this licence, visit http://creativecommons.org/licenses/by/4.0/.

\section{References}

Papers of particular interest, published recently, have been highlighted as:

- Of importance

•- Of major importance

1. Ponikowski P, Voors AA, Anker SD, Bueno H, Cleland JGF, Coats AJS, et al. 2016 ESC guidelines for the diagnosis and treatment of acute and chronic heart failure: the task force for the diagnosis and treatment of acute and chronic heart failure of the European Society of Cardiology (ESC) developed with the special contribution of the heart failure association (HFA) of the ESC. Eur Heart J. 2016;37: 2129-200.

2. Chen S, Hsu W-Y, Lin Y-N, Wang C-Y, Wu C-H, Chang K-H. Incidence and risk of major adverse cardiovascular events in middle-aged patients with chronic kidney disease: a populationbased cohort study. Int Urol Nephrol. 2019;51:1219-27.

3. Damman K, Valente MAE, Voors AA, O'Connor CM, van Veldhuisen DJ, Hillege HL. Renal impairment, worsening renal function, and outcome in patients with heart failure: an updated meta-analysis. Eur Heart J. 2014;35:455-69.

4. Streng KW, Nauta JF, Hillege HL, Anker SD, Cleland JG, Dickstein K, et al. Non-cardiac comorbidities in heart failure with reduced, mid-range and preserved ejection fraction. Int J Cardiol. 2018;271:132-9.

5. Lawson CA, Testani JM, Mamas M, Damman K, Jones PW, Teece L, et al. Chronic kidney disease, worsening renal function and outcomes in a heart failure community setting: a UK national study. Int J Cardiol. 2018;267:120-7. 
6. Bansal N, Zelnick L, Bhat Z, Dobre M, He J, Lash J, et al. Burden and outcomes of heart failure hospitalizations in adults with chronic kidney disease. J Am Coll Cardiol. 2019;73:2691-700.

7. Hakopian NN, Gharibian D, Nashed MM. Prognostic impact of chronic kidney disease in patients with heart failure. Perm J. 2019;23. https://doi.org/10.7812/TPP/18.273. This study examined impact of CKD in patients with $\mathrm{HF}$ and demonstrated that stage 4 and $5 \mathrm{CKD}$ is a significant contributor to poor prognosis in patients with HF, leading to significantly higher rates of hospitalization, 30-day readmission, and mortality.

8. ter Maaten JM, Damman K, Verhaar MC, Paulus WJ, Duncker DJ, Cheng $\mathrm{C}$, et al. Connecting heart failure with preserved ejection fraction and renal dysfunction: the role of endothelial dysfunction and inflammation. Eur J Heart Fail. 2016;18:588-98.

9. AA H, Wanner C, Sarnak MJ, Piña IL, McIntyre CW, Komenda P, et al. Heart failure in chronic kidney disease: conclusions from a kidney disease: improving global outcomes (KDIGO) controversies conference. Kidney Int. 2019;95:1304-17.

10. Unger ED, Dubin RF, Deo R, Daruwalla V, Friedman JL, Medina $\mathrm{C}$, et al. Association of chronic kidney disease with abnormal cardiac mechanics and adverse outcomes in patients with heart failure and preserved ejection fraction. Eur J Heart Fail. 2016;18:103-12.

11. He J, Shlipak M, Anderson A, Roy JA, Feldman HI, Kallem RR, et al. Risk factors for heart failure in patients with chronic kidney disease: the CRIC (chronic renal insufficiency cohort) study. J Am Heart Assoc. 2017 May; 17:6.

12. https://www.nice.org.uk/guidance/cg182/chapter/1Recommendations\#pharmacotherapy. Last Accessed 3.11.2019.

13. Hein AM, Scialla JJ, Edmonston D, Cooper LB, DeVore AD, Mentz RJ. Medical management of heart failure with reduced ejection fraction in patients with advanced renal disease. JACC Heart Fail. 2019;7:371-82.

14. Haynes R, Zhu D, Judge PK, Herrington WG, Kalra PA, Baigent C. Chronic kidney disease, heart failure and neprilysin inhibition. Nephrol Dial Transplant. 2019. https://doi.org/10.1093/ndt/gfz058.

15. Piepoli MF, Hoes AW, Agewall S, Albus C, Brotons C, Catapano AL, et al. 2016 European Guidelines on cardiovascular disease prevention in clinical practice. Eur Heart J. 2016;37:2315-81.

16. Ribic CM, Holland D, Howell J, Jevnikar A, Kim SJ, Knoll G, et al. Study of cardiovascular outcomes in renal transplantation: a prospective, multicenter study to determine the incidence of cardiovascular events in renal transplant recipients in Ontario, Canada. Can J Kidney Health Dis. 2017;4:2054358117713729.

17. Methven S, Steenkamp R, Fraser S. UK renal registry 19th annual report: chapter 5 survival and causes of death in UK adult patients on renal replacement therapy in 2015: national and centre-specific analyses. Nephron. 2017;137(Suppl 1):117-50.

18. Saran R, Robinson B, Abbott KC, Agodoa LYC, Bragg-Gresham J, Balkrishnan R, et al. US renal data system 2018 annual data report: epidemiology of kidney disease in the United States. Am J Kidney Dis. 2019;73:A7-8.

19. Ohyama Y, Ambale-Venkatesh B, Noda C, Kim J-Y, Tanami Y, Teixido-Tura G, et al. Aortic arch pulse wave velocity assessed by magnetic resonance imaging as a predictor of incident cardiovascular events: the MESA (multi-ethnic study of atherosclerosis). Hypertension. 2017;70:524-30.

20. Adenwalla SF, Graham-Brown MPM, Leone FMT, Burton JO, McCann GP. The importance of accurate measurement of aortic stiffness in patients with chronic kidney disease and end-stage renal disease. Clin Kidney J. 2017;10:503-15. This review discusses the advantages and limitations of non-invasive methods that have been used to assess aortic stiffness, and analyze the relationship between aortic stiffness and cardiovascular health in patients with renal disease.
21. Hayer MK, Price AM, Liu B, Baig S, Ferro CJ, Townend JN, et al. Diffuse myocardial interstitial fibrosis and dysfunction in early chronic kidney disease. Am J Cardiol. 2018;121:656-60. This study demonstrated in patients with early-stage CKD that noninvasive imaging biomarkers of myocardial fibrosis do not progress if renal function remains stable.

22. Graham-Brown MPM, March DS, Churchward DR, Stensel DJ, Singh A, Arnold R, et al. Novel cardiac nuclear magnetic resonance method for noninvasive assessment of myocardial fibrosis in hemodialysis patients. Kidney Int. 2016;90:835-44.

23. Chen M, Arcari L, Engel J, Freiwald T, Platschek S, Zhou H, et al. Aortic stiffness is independently associated with interstitial myocardial fibrosis by native $\mathrm{T} 1$ and accelerated in the presence of chronic kidney disease. Int J Cardiol Heart Vasc. 2019;24:100389. The study demonstrated strong relation between aortic stiffness and myocardial hypertrophic-fibrotic remodeling in CKD. There was no such relation in the non-CKD cohort despite similar CV risk profile as CKD patients.

24. Temimović R, Rašić S, Džubur A. Cardiovascular remodelling in patients with pre-dialysis chronic kidney disease and renal transplant recipients. Med Glas (Zenica). 2019;16(2). https://doi.org/10. 17392/1009-19. This study evaluated the relationship between cardiovascular remodelling and glomerular filtration rate in pre-dialysis chronic kidney disease patients without cardiovascular diseases and in renal transplant recipients.

25. Tamulènaitė E, Žvirblyte R, Ereminienẻ R, Žiginskienè E, Ereminiené E. Changes of left and right ventricle mechanics and function in patients with end-stage renal disease undergoing haemodialysis. Medicina (Kaunas). 2018;13;54(5). https://doi.org/ 10.3390/medicina54050087.

26. Matsuo H, Dohi K, Machida H, Takeuchi H, Aoki T, Nishimura H, et al. Echocardiographic assessment of cardiac structural and functional abnormalities in patients with end-stage renal disease receiving chronic hemodialysis. Circ J. 2018;82:586-95.

27. Peng W, Li Z, Xu H, Xia C, Guo Y, Zhang J, et al. Assessment of right ventricular dysfunction in end-stage renal disease patients on maintenance haemodialysis by cardiac magnetic resonance imaging. Eur J Radiol. 2018;102:89-94.

28. Xhakollari L, Leosdottir M, Magnusson M, Holzmann MJ, Nilsson PM, Christensson A. Echocardiographic findings in patients with mild to moderate chronic kidney disease without symptomatic heart failure: a population-based study. Cardiorenal Med. 2019;9:284296. https://doi.org/10.1159/000499835.

29. Altmann U, Böger CA, Farkas S, Mack M, Luchner A, Hamer OW, et al. Effects of reduced kidney function because of living kidney donation on left ventricular mass. Hypertension. 2017;69:297-303.

30. Moody WE, Ferro CJ, Edwards NC, Chue CD, ELS L, Taylor RJ, et al. Cardiovascular effects of unilateral nephrectomy in living kidney donors. Hypertension. 2016;67:368-77. The study demonstrated that one of the earliest signs of subclinical cardiac involvement of CKD are increased $L V$ mass and increased aortic stiffness.

31. Weidner K, Behnes M, Weiß C, Nienaber C, Reiser L, Bollow A, et al. Impact of chronic kidney disease on recurrent ventricular tachyarrhythmias in ICD recipients. Heart Vessels. 2019. Heart Vessels. 2019;34:1811-1822. https://doi.org/10.1007/s00380-01901415-z.

32. Puntmann VO, Valbuena S, Hinojar R, Petersen SE, Greenwood JP, Kramer CM, et al. Society for cardiovascular magnetic resonance (SCMR) expert consensus for CMR imaging endpoints in clinical research: part I - analytical validation and clinical qualification. J Cardiovasc Magn Reson. 2018;20:67.

33. Reiter T, Ritter O, Prince MR, Nordbeck P, Wanner C, Nagel E, et al. Minimizing risk of nephrogenic systemic fibrosis in cardiovascular magnetic resonance. J Cardiovasc Magn Reson. 2012;14: 31. https://doi.org/10.1186/1532-429X-14-31. 
34. https:/www.ema.europa.eu/en/medicines/human/referrals/ gadolinium-containing-contrast-agents. Last Accessed 1.11.2019.

35. Nagel E, Greenwood JP, McCann GP, Bettencourt N, Shah AM, Hussain ST, et al. Magnetic resonance perfusion or fractional flow reserve in coronary disease. N Engl J Med. 2019;380:2418-28.

36. Puntmann VO, Elif P, Chandrashekhar Y, Eike N. T1 mapping in characterizing myocardial disease. Circ Res. 2016;119:277-99.

37. Rutherford E, Weir-McCall JR, Patel RK, Houston JG, Roditi G, Struthers AD, et al. Research cardiac magnetic resonance imaging in end stage renal disease-incidence, significance and implications of unexpected incidental findings. Eur Radiol. 2017;27:315-24. The study demonstrated that patients with CKD had higher incidence of noncardiac findings, which are clinically significant in approximately $15 \%$ and can be reliably diagnosed using CMR imaging.

38. Rutherford E, Talle MA, Mangion K, Bell E, Rauhalammi SM, Roditi $\mathrm{G}$, et al. Defining myocardial tissue abnormalities in endstage renal failure with cardiac magnetic resonance imaging using native T1 mapping. Kidney Int. 2016;90:845-52.

39. Graham-Brown MPM, Rutherford E, Levelt E, March DS, Churchward DR, Stensel DJ, et al. Native T1 mapping: inter-study, inter-observer and inter-center reproducibility in hemodialysis patients. J Cardiovasc Magn Reson. 2017;19:21. The study highlights the fact that there is no significant correlation between change in native $\mathrm{T} 1$ values and change in body weight of hemodialysis patients, who underwent repeated CMR scan at a fixed interval from dialysis.

40. Kotecha T, Martinez-Naharro A, Yoowannakul S, Lambe T, Rezk $\mathrm{T}$, Knight DS, et al. Acute changes in cardiac structural and tissue characterisation parameters following haemodialysis measured using cardiovascular magnetic resonance. Sci Rep. 2019;9:1388.

41. Arcari L, Hinojar R, Engel J, Freiwald T, Platschek S, Zainal H, et al. Native $\mathrm{T} 1$ and $\mathrm{T} 2$ provide distinctive signatures in hypertrophic cardiac conditions - Comparison of uremic, hypertensive and hypertrophic cardiomyopathy. Int J Cardiol. 2020;306:102-8. https:// doi.org/10.1016/j.ijcard.2020.03.002. This study revealed different CMR signatures of common hypertrophic cardiac phenotypes. Native T1 was raised in all conditions, indicating the presence of pathologic hypertrophic remodelling. Markedly raised native $T 2$ was $C K D$-specific, suggesting a prominent role of intramyocardial fluid.

42. Hayer MK, Radhakrishnan A, Price AM, Baig S, Liu B, Ferro CJ, et al. Early effects of kidney transplantation on the heart-a cardiac magnetic resonance multi-parametric study. Int J Cardiol. 2019;293:272-7.

43. Hamidi S, Kojuri J, Attar A, Roozbeh J, Moaref A, Nikoo MH. The effect of kidney transplantation on speckled tracking echocardiography findings in patients on hemodialysis. J Cardiovasc Thorac Res. 2018;10:90-4.

44. Gong IY, Al-Amro B, Prasad GVR, Connelly PW, Wald RM, Wald $\mathrm{R}$, et al. Cardiovascular magnetic resonance left ventricular strain in end-stage renal disease patients after kidney transplantation. J Cardiovasc Magn Reson. 2018;20:83.

45. Ohyama Y, Ambale-Venkatesh B, Noda C, Chugh AR, TeixidoTura G, Kim J-Y, et al. Association of aortic stiffness with left ventricular remodeling and reduced left ventricular function measured by magnetic resonance imaging: the multi-ethnic study of atherosclerosis. Circ Cardiovasc Imaging. 2016;9(7). https://doi. org/10.1161/CIRCIMAGING.115.004426. The study showed that significantly increased aortic stiffness is associated with $L V$ remodeling, reduced $L V$ systolic and diastolic function.

46. Puntmann VO, Arroyo Ucar E, Hinojar Baydes R, Ngah NB, Kuo Y-S, Dabir D, et al. Aortic stiffness and interstitial myocardial fibrosis by native $\mathrm{T} 1$ are independently associated with left ventricular remodeling in patients with dilated cardiomyopathy. Hypertension. 2014;64:762-8.

47. Cheddani L, Radulescu C, Chaignon M, Karras A, Neuzillet Y, Duong J-P, et al. From arterial stiffness to kidney graft microvasculature: mortality and graft survival within a cohort of 220 kidney transplant recipients. PLoS One. 2018;13:e195928. The study showed no significant changes in aortic stiffness observed in the first year after renal transplantation.

48. Odudu A, Eldehni MT, McCann GP, Horsfield MA, Breidthardt T, McIntyre CW. Characterisation of cardiomyopathy by cardiac and aortic magnetic resonance in patients new to hemodialysis. Eur Radiol. 2016;26:2749-61.

49. Parikh JD, Hollingsworth KG, Kunadian V, Blamire A, MacGowan GA. Measurement of pulse wave velocity in normal ageing: comparison of Vicorder and magnetic resonance phase contrast imaging. BMC Cardiovasc Disord. 2016;16:50.

50. Puntmann VO, Nagel E, Hughes AD, Gebker R, Gaddum N, Chowienczyk P, Jahnke C, Mirelis J, Schnackenburg B, Paetsch I, Fleck E. Gender-specific differences in myocardial deformation and aortic stiffness at rest and dobutamine stress..Hypertension. 2012;59(3):712-8.

51. Salvi P, Scalise F, Rovina M, Moretti F, Salvi L, Grillo A, et al. Noninvasive estimation of aortic stiffness through different approaches. Hypertension. 2019;74:117-29.

52. Grillo A, Parati G, Rovina M, Moretti F, Salvi L, Gao L, et al. Shortterm repeatability of noninvasive aortic pulse wave velocity assessment: comparison between methods and devices. Am J Hypertens. 2017;31:80-8.

53. Graham-Brown MPM, Adenwalla SF, Lai FY, Hunt WH, Parke K, Gulsin $\mathrm{G}$, et al. The reproducibility of cardiac magnetic resonance imaging measures of aortic stiffness and their relationship to cardiac structure in prevalent haemodialysis patients. Clin Kidney J. 2018;11:864-73.

54. Winau L, Hinojar Baydes R, Braner A, Drott U, Burkhardt H, Sangle S, et al. High-sensitive troponin is associated with subclinical imaging biosignature of inflammatory cardiovascular involvement in systemic lupus erythematosus. Ann Rheum Dis. 2018;77(11):1590-98. https://doi.org/10.1136/annrheumdis-2018213661. This study revealed an important link between myocardial inflammation and injury, which is amplified through increased PVW.

Publisher's Note Springer Nature remains neutral with regard to jurisdictional claims in published maps and institutional affiliations. 